\title{
Central serous chorioretinopathy in a patient with Flammer Syndrome
}

\author{
Johann Gauer ${ }^{1}$, Lei Fang ${ }^{2,3}$, Maneli Mozaffarieh ${ }^{1,2,4}$
}

\section{ABSTRACT}

Background: Patients with Flammer Syndrome (FS) may suffer from central serous chorioretinopathy (CSCR). Treatment of FS-associated vascular dysregulation can lead to stabilization and a promising outcome in such patients.

Case summary: A 57-year old woman presented in our clinic with a blurred vision in her right eye for a week. Vascular anamnesis showed that the patient frequently suffered from cold hands and feet, tinnitus, migraines, a low blood pressure, and had a reduced feeling of thirst. Anterior segment and intraocular pressure values of $13 \mathrm{~mm} \mathrm{Hg}(\mathrm{OD})$ and $16 \mathrm{~mm} \mathrm{Hg}(\mathrm{OS})$ were normal in both eyes. The retinal venous pressure was increased to $23 \mathrm{~mm} \mathrm{Hg}$ in the right eye and $25 \mathrm{~mm} \mathrm{Hg}$ in the left eye. Posterior examination showed a serous retinal detachment of the right eye.

Conclusion: The patient's condition improved four months after treatment of vascular dysregulation showing that FS may be a cause for CSCR and therefore in such cases it is worth treating FS.

Keywords: central serous chorioretinopathy, Flammer Syndrome, vascular dysregulation, retinal venous pressure, serous retinal detachment

\section{INTRODUCTION}

Patients with central serous chorioretinopathy (CSCR) develop serous retinal detachment and/or retinal pigment epithelial detachment, particularly in the area of the macula, due to leakage of fluid through the retinal pigment epithelium into the subretinal space. ${ }^{1,2}$ Although the pathogenesis of this disease remains unknown, previous reports suggest an association of CSCR with the vascular dysregulation in the context of the Flammer Syndrome (FS) (Figure 1) ${ }^{3,4} \mathrm{FS}$ is a benign and prevalent condition describing the phenotype of people who are generally healthy but have an altered reaction of blood vessels to stimuli such as cold or emotional stress. ${ }^{5,6}$ Similar to people with FS, who suffer from a primary vascular dysregulation ${ }^{7,8}$, CSCR patients display a local vascular dysfunction of the of the choroidal veins. ${ }^{3}$ In both FS and in the acute phase of CSCR, plasma endothelin (ET-1) levels are increased. ${ }^{9,10}$ As a result, retinal venous pressure measured by means of ophthalmodynamometry is increased in FS patients. ${ }^{9,10}$
${ }^{1}$ Medix Gruppen Praxis, Zurich, Switzerland 'University of Basel, Basel, Switzerland ${ }^{3}$ Department of Biomedicine, University Hospital Basel, Switzerland

${ }^{4}$ Limmat Eye Center, Zurich, Switzerland

Corresponding author:

PD Dr med Maneli Mozaffarieh

University of Basel \& Limmat-Eye Center, Zürich

Hardturmstrasse 133, 8005 Zürich

E-mail: maneli.mozaffarieh@gmail.com

\section{DOl: 10.36000/hbT.2021.01.002}

ISSN: 2673-8406 (Print) and 2673-8414 (Online)

Gauer J et al. Central serous chorioretinop athy in a patient with Flammer Syndrome [published online ahead of print January 30 , 2021]. hbT Schw Aerztej. 2021; 1: 82-84. 


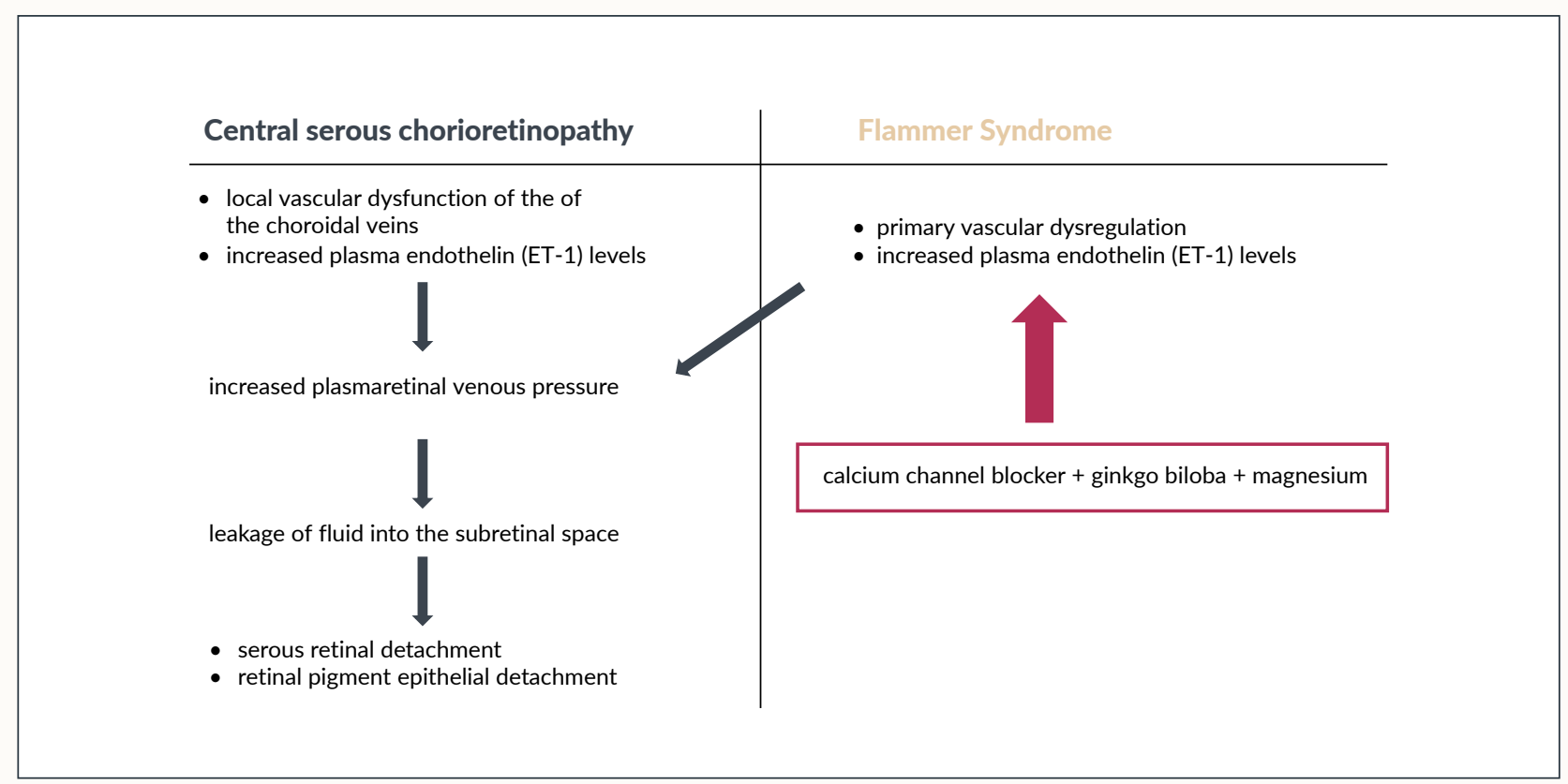

Figure 1. Association of the pathogenesis between central serous chorioretinopathy (left) and Flammer Syndrome (right). ET-1, endothelin-1.

\section{CASE PRESENTATION}

\section{CLINICAL FINDINGS}

A 57-year old woman presented with blurring of vision, cloudiness, and a dark spot on her right eye for a week. She has not been using any systemic or local corticosteroid medication but stated that she had suffered from emotional stress. Vascular anamnesis indicated that the patient suffered from FS: her hands and feet were frequently cold even during summer, she had low blood pressure, reduced feeling of thirst (she had to remind herself to drink enough fluid), and she suffered from migraines and at times from tinnitus. Her best corrected visual acuity was 0.8 in the right eye and 1.0 in the left eye. Anterior segment and intraocular pressure of $13 \mathrm{~mm}$ $\mathrm{Hg}$ (right eye) and $16 \mathrm{~mm} \mathrm{Hg}$ (left eye) was normal. Retinal venous pressure was increased in both eyes to $23 \mathrm{~mm} \mathrm{Hg}$ in the right eye and $25 \mathrm{~mm} \mathrm{Hg}$ in the left eye (lack of a spontaneous retinal vein pulsation). Posterior segment examination of her right eye showed a serous retinal detachment. There were no signs of intraocular inflammation in either eye. The left eye showed normal posterior segment findings.

\section{TREATMENT}

Treatment aimed at the improvement of the vascular dysregulation in the context of FS. The patient received a low dosed calcium channel blocker ( 4 drops Nifedipine in a liter of water daily, ginkgo biloba (120 mg daily) and magnesium (12 mmol daily). At her four-months return visit, the best corrected visual acuity measured 20/20 in both eyes, retinal venous pressure values had basically normalized to $18 \mathrm{~mm} \mathrm{Hg}$ in the right eye and $17 \mathrm{~mm} \mathrm{Hg}$ in the left eye (IOP right eye: 14 $\mathrm{mm} \mathrm{Hg}$, IOP left eye: $12 \mathrm{~mm} \mathrm{Hg}$ ), and the posterior segment showed a resolution of the subretinal fluid. 


\section{DISCUSSION}

CSCR is characterized by an accumulation of subretinal fluid in the macula caused by abnormalities of the choroidal circulation. ${ }^{2}$ Although the disease generally affects the male population in their third and fourth decades, ${ }^{11}$ females may be affected as well. While the cause of CSCR remains unknown, several risk factors have been reported. In particular, the use of glucocorticoids ${ }^{12}$ and stressful events associated with higher endogenous corticosteroid levels have been correlated to the development of CSCR. Type A personality has also been reported to be associated with CSCR, ${ }^{13}$ along with other potential risk factors including us of tobacco, alcohol, sympathomimetic medication or psychotropic medication and autoimmune disorders. ${ }^{14}$

Abnormalities in the choroidal circulation play an important role in the pathogenesis of CSCR. Choroidal blood flow changes can be induced through enhanced oxidative stress, inflammatory cells and nitric oxide. ${ }^{7}$ The hyperpermeable choroidal vessels increase tissue hydrostatic pressure, leading to the breakdown of the blood retinal barrier. ${ }^{15}$ This in turn promotes pigment epithelial detachment and subretinal fluid accumulation. Back in 1996, when ocular blood flow measurements were performed in CSCR patients, C Prünte and J Flammer found that the patients had a delayed arterial filling followed by capillary and venous congestion. ${ }^{2}$ These observations support an involvement of primary vascular dysregulation in the context of the FS in these patients.

In short, although the origins of CSCR are yet unknown and various therapeutic options may be useful for individual patients, in patients with confirmed FS, treatment of vascular dysregulation may prevent CSCR or mitigate the course of the disease. Knowledge about the association of CSCR with FS may thus be useful for general practitioners, who tend to see the patients first.

\section{TAKE-HOME MESSAGES}

- Patients with FS may suffer from CSCR due to FS-associated vascular dysregulation.

- Treatment of the FS-associated vascular dysregulation can attenuate or resolve CSCR.

\section{CONFLICT OF INTEREST}

None.

\section{AUTHOR CONTRIBUTIONS}

MM took the lead in writing the manuscript, JG made substantial contributions to the design, acquisition of data and presentation, LF contributed to the final version of the manuscript, provided critical feed-back and inserted references.

\section{FUNDING}

None.

\section{ACKNOWLEDGEMENT}

None.

1. Semeraro F et al. Central Serous Chorioretinopathy: Pathogenesis and Management. Clin Ophthalmol. 2019; 13(12): 2341-52.

2. Nicholson B et al. Central serous chorioretinopathy: update on pathophysiology and treatment. Surv Ophthalmol. 2013: 58(2): 103-26.

3. Prunte $\mathrm{C}$ et al. Choroidal capillary and venous congestion in central serous chorioretinopathy. Am J Ophthalmol. 1996; 121(1): 26-34.

4. Josifova T et al. Flammer Syndrome, A Potential Risk Factor for Central Serous Chorioretinopathy. Biomed J Sci \& Tech Res. 2020; 24(2): 18115-9.

5. Vahedian $Z$ et al. Flammer Syndrome and glaucoma. healthbook TIMES, 2020; (1): 2-6.

\author{
6. Konieczka K et al. Flammer syndrome. EPMA J. 2014; \\ 5(1): 11 . \\ 7. Mozaffarieh $\mathrm{M}$ et al. Oxygen and blood flow: players \\ in the pathogenesis of glaucoma. Mol Vis. 2008; 14: \\ $224-2$ \\ 8. Flammer $\mathrm{J}$ et al. Autoregulation, a balancing act \\ between supply and demand. Can J Ophthalmol. 2008 \\ 43(3): $317-21$ \\ 9. Kida T et al. Data on the involvement of endothelin-1 \\ (ET-1) in the dysregulation of retinal veins. Data Brief. \\ 2018; 21: 59-62. \\ 10. Türkçüoğlu $P$ et al. The role of serum endothelin-1 \\ level in the etiopathogenesis of central serous chorioretin- \\ opathy. Am J Ophthalmol. 2006; 142(2): 349-51.
}

11. Kitzmann AS et al. The incidence of central serous chorioretinopathy in Olmsted County, Minnesota, 1980-2002. Ophthalmology, 2008; 115(1): 169-73.

12. Nicholson $B P$ et al. Central serous chorioretinopathy and glucocorticoids: an update on evidence for association. Surv Ophthalmol. 2018; 63(1): 1-8.

13. Yannuzzi LA. Type-A behavior and central serous chorioretinopathy. Retina, 1987; 7(2): 111-31.

14. Liu B et al. Risk Factors for Central Serous Chorioretinopathy: A Systematic Review and Meta-Analysis. Retina. 2016; 36(1): 9-19.

15. Cunha-Vaz J. The blood-ocular barriers. Surv Ophthalmol. 1979; 23(5): 279-96. 